\title{
Progressive multifocal leukoencephalopathy developing in a fludarabine naïve patient with chronic lymphocytic leukemia
}

\author{
Melissa G Ooi' \\ Peter J Kelly ${ }^{2}$ \\ Michael Farrell ${ }^{3}$ \\ Philip Murphy ${ }^{4}$ \\ Peter O'Gorman' \\ 'Department of Hematology; \\ ${ }^{2}$ Department of Neurology, Mater \\ Misericordiae Hospital, Dublin, Ireland; \\ ${ }^{3}$ Department of Neuropathology; \\ ${ }^{4}$ Department of Hematology, \\ Beaumont Hospital, Dublin, Ireland
}

Correspondence: Melissa G Ooi

Department of Hematology, Mater Misericordiae Hospital, Eccles Street, Dublin I, Ireland

Tel +353 I 8034412

Emailmgooi2077@yahoo.co.uk

\begin{abstract}
We report a case of progressive multifocal leukoencephalopathy developing in a fludarabine-naïve patient with chronic lymphocytic leukemia. We suggest that progressive multifocal leukoencephalopathy should be considered in any chronic lymphocytic leukemia patient with unexplained neurological symptoms. Treatment history does not necessarily require fludarabine. Intrathecal cytosine arabinoside although unsuccessful in this patient, is a therapeutic option to consider in patients experiencing side effects from systemic therapy.
\end{abstract}

Keywords: progressive multifocal leukoencephalopathy, chronic lymphocytic leukemia, JC virus, fludarabine, intrathecal cytosine arabinoside

\section{Case report}

A 55-year-old Caucasian man presented with progressive visual impairment. He had difficulty in following words on a printed page and was 'jumping from one line to the next'. This was associated with occipital headaches. He had no other neurological symptoms. Neurological examination was normal.

Past medical history was significant for chronic lymphocytic leukemia (CLL) diagnosed 15 years ago. Since his diagnosis he had received several courses of chlorambucil and corticosteroids with good response each time. In fact, he received three courses of chlorambucil and prednisolone in the year prior to these visual symptoms. There was no significant other past medical history or family history. Magnetic resonance imaging (MRI) of the brain revealed a lesion in the occipital lobe. Other investigations including repeated cerebrospinal fluid analysis were not conclusive. The patient refused a brain biopsy.

Three months later, the patient's vision was progressively worsening with new dysarthria and weakness of his right arm and right leg affecting his gait and balance. The patient finally consented and a stereotactic brain biopsy of the lesion in the occipital lobe was carried out.

The biopsy showed myelin loss and astrocytic atypia with occasional inclusions (Figures 1 and 2). Immunohistochemistry was positive for John Cunningham (JC) virus with the COL-5 strain antibody. In-situ hybridization for JC virus was positive. These findings are diagnostic of progressive multifocal leukoencephalopathy (PML).

Therapy for PML was then commenced. He was initially started on intravenous cidofivir. After two courses, his neurologic signs continued to progress through treatment and he was switched to intravenous cytosine arabinoside $2 \mathrm{mg} / \mathrm{kg}$ per day for five days as described by Aksamit. ${ }^{1}$ He received two cycles of intravenous cytosine arabinoside, but this was complicated by neutropenic sepsis from the systemic therapy. He was then switched to intrathecal liposomal cytarabine (x one cycle). The rationale for the intrathecal therapy was to deliver treatment directly to the central 


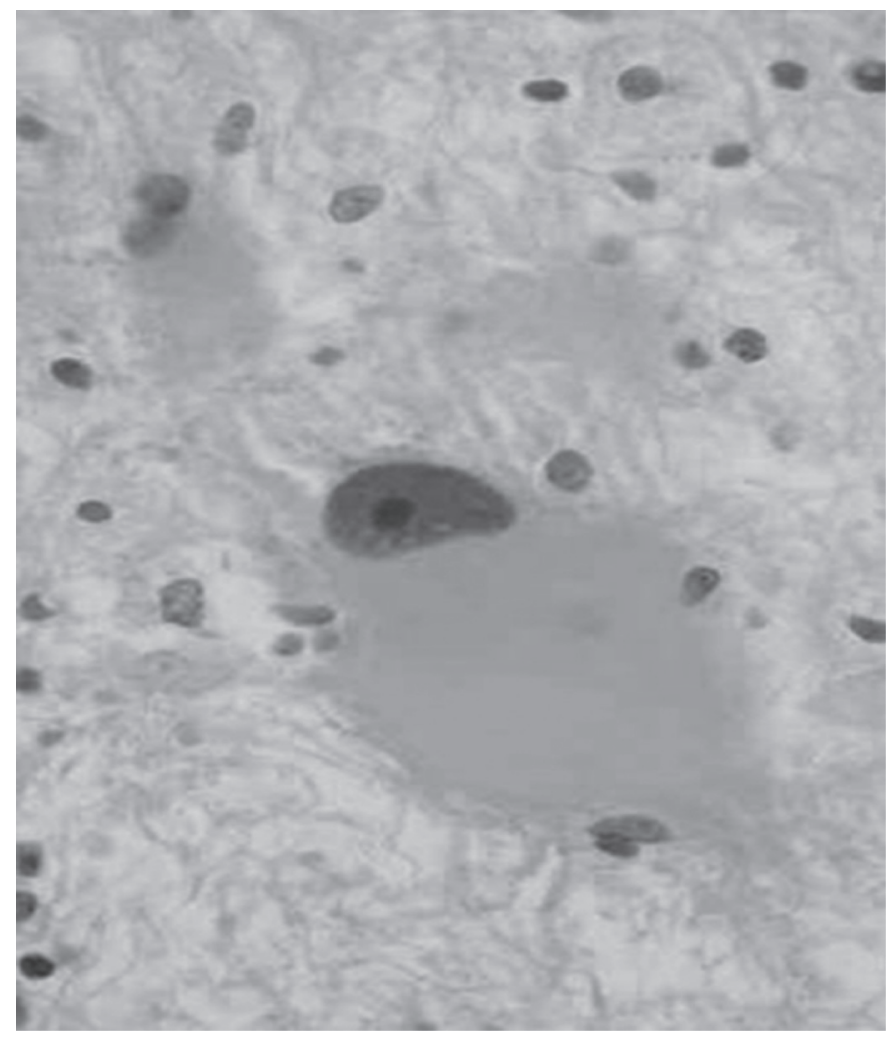

Figure I Brain biopsy showing changes suggestive of PML and large abnormally hypertrophied reactive astrocytes are visible.

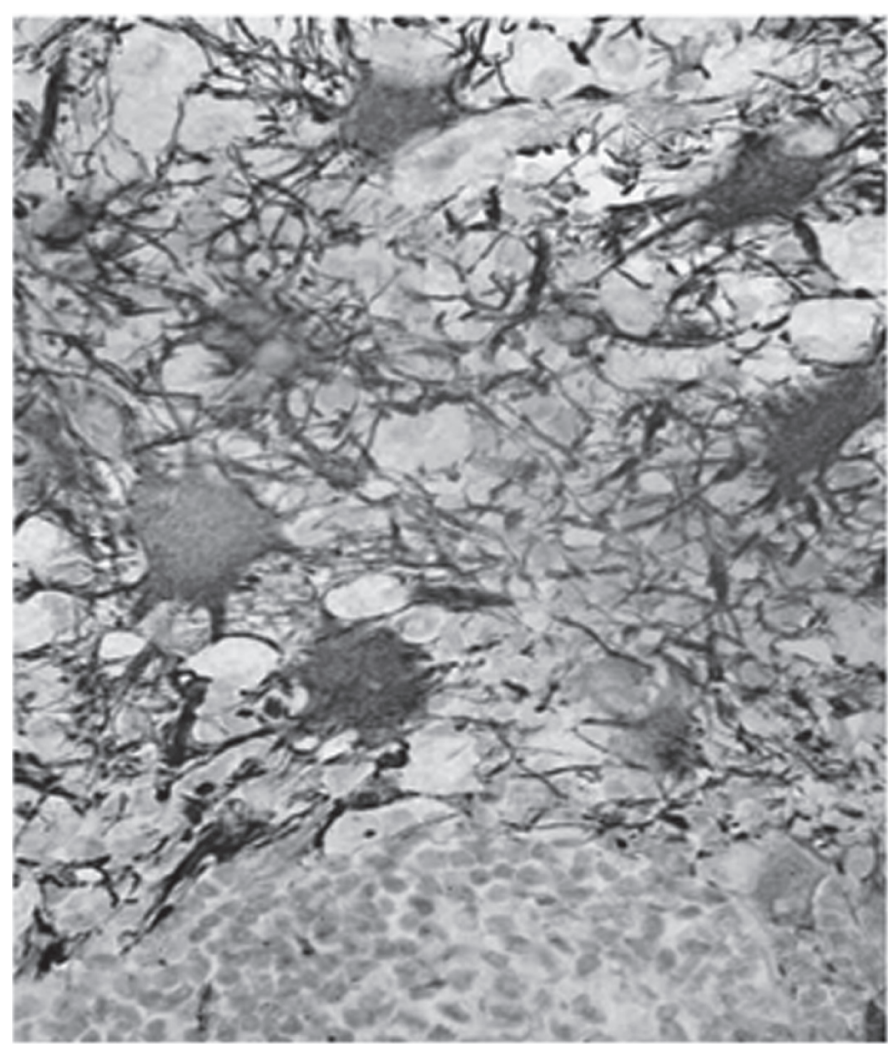

Figure $\mathbf{2}$ The large hypertrophied astrocytes stain positive with glial fibrillary acidic protein. 
nervous system and minimize systemic toxicity. There was progressive deterioration despite treatment; the patient was unable to communicate and was paraplegic. The decision to discontinue further treatment was made in conjunction with the family and he died six months later.

\section{Discussion}

Opportunistic infections are a major cause of morbidity and mortality in CLL patients. It is estimated that up to $80 \%$ of CLL patients will develop infectious complications at some time in their disease course, with infections accounting for up to $60 \%$ of deaths. ${ }^{2}$ The pathogenesis of infection in CLL patients is multifactorial, involving a variety of immune defects inherent to the disease process and further influenced by disease stage and subsequent therapy with immunosuppressive agents.

PML is an opportunistic viral infection resulting from impaired cell mediated immune response. It is caused by either a primary infection or a secondary reactivation in the brain oligodendrocytes of the JC virus. Thus PML has been first recognized as a late complication of various chronic diseases such as leukemia, lymphoma, sarcoidosis, and carcinomatosis. With increasing use of immunosuppressive agents in the treatment of autoimmune diseases and organ transplantation, PML has also appeared in these settings. HIV infection has become the major risk factor for the development of PML due to acquired impairment of cell-mediated immunity.

There is no causal link with a specific immunosuppressant drug. Corticosteroids, cyclophosphamide, chlorambucil, azathioprine and cyclosporin A have all been associated with the occurrence of PML in case reports in the setting of rheumatologic and hematologic malignancy. There have been many case reports published linking PML with CLL. The therapeutic agents used in most cases described used combination chemotherapy and/or fludarabine.

There are only two other cases, similar to our case, reported that associate treatment with alkylating agents alone with PML. ${ }^{3,4}$ There are two additional cases that are not associated with any treatment. ${ }^{5,6}$ The fact that these patients had no previous treatment lends support to the fact that CLL is by itself immunosuppressive as a disease process.

There is no data in the literature on the significance of the interval between diagnosis of CLL and presentation of PML. It could be speculated that the longer the duration of immunosuppression, the higher the risk that the JC virus will be reactivated.

No effective treatment is available at present. Anecdotal reports show some efficacy of cidofovir, interferon alfa, and cytosine arabinoside. The median survival for PML in combination with CLL is 4.3 months. ${ }^{7}$

\section{Acknowledgments}

I would like to thank the patient and his family for their consent to the publication of this case report. The authors report no conflicts of interest in this work.

\section{References}

1. Aksamit AJ. Treatment of non-AIDS progressive multifocal leukoencephalopathy with cytosine arabinoside. J Neurovirol. 2001;7:386-90.

2. Morrison VA. The infectious complications of chronic lymphocytic leukaemia. Semin Oncol. 1998;25:98-106.

3. Bagnato F, Pietropoaolo V, Di Taranto C, Lorenzano S, Toni D. Chronic lymphocytic leukemia complicated by progressive multifocal leukoencephalopathy without apparent immunodepression. Eur J Neurol. 2001;8:367-368.

4. Farge D, Herve R, Mikol J, et al. Simultaneous progressive multifocal leukoencephalopathy, Ebstein-Barr virus (EBV) latent infection and cerebral parenchymal infiltration during chronic lymphocytic leukaemia. Leukemia. 1994;8(2):318-321.

5. Nowak-Michalska T, Barcikowska M, Kida E, Budka H, Liberski PP A case of progressive multifocal leucoencephalopathy during chronic lymphocytic leukaemia. Neurol Neurochir Pol. 1993;27:905-912.

6. O'Riordan S, McGuigan C, Farrell M, Hutchinson M. Progressive multifocal leucoencephalopathy presenting with parkinsonism. J Neurol. 2003;250:1379-1381.

7. Holzapfel C, Kellinghaus C, Luttmann R, et al. Progressive multifocal leukoencephalopathy (PML) in chronic lymphatic leukemia (CLL). Review of the literature and case report. Nervenarzt. 2002;73:543-547. 
